\title{
A NOTE ON THE UNCLOUDING THE SKY OF NEGATIVELY CURVED MANIFOLDS
}

\author{
ALBERT BORBÉLY
}

(Received 15 August 2007)

\begin{abstract}
The problem of finding geodesics that avoid certain obstacles in negatively curved manifolds has been studied in different situations. In this note we give a generalization of the unclouding theorem of J. Parkkonen and F. Paulin: there is a constant $s_{0}=1.534$ such that for any Hadamard manifold $M$ with curvature $\leq-1$ and for any family of disjoint balls or horoballs $\left\{C_{a}\right\}_{a \in A}$ and for any point $p \in M-\bigcup_{a \in A} C_{a}$ if we shrink these balls uniformly by $s_{0}$ one can always find a geodesic ray emanating from $p$ that avoids the shrunk balls. It will be shown that in the theorem above one can replace the balls by arbitrary convex sets.
\end{abstract}

1991 Mathematics subject classification: 53C22.

Keywords and phrases: convex sets, negative curvature, geodesics.

\section{Introduction}

The problem of finding geodesics that avoid certain obstacles in negatively curved manifolds has been studied in different situations. It found applications in several areas like the existence of bounded geodesics in manifolds with finite volume [3-5] and the construction of proper closed invariant subsets of the unit tangent bundle with large footprints $[1,6]$.

An interesting theorem [4, Theorem 1.1] of this kind was proved recently by Parkkonen and Paulin for $C A T(-1)$ metric spaces (an earlier version of the theorem appeared in [3]). The statement in the Riemannian setting is as follows.

The unclouding theorem (J. Parkkonen and F. Paulin). There is a constant $s_{0}=1.534$ such that for any Hadamard manifold $M$ with curvature $\leq-1$ and for any family of disjoint balls or horoballs $\left\{C_{a}\right\}_{a \in A}$ and for any point $p \in M-\bigcup_{a \in A} C_{a}$ if we shrink these balls uniformly by $s_{0}$ one can always find a geodesic ray emanating from $p$ that avoids the shrunk balls.

Our goal is to show that in the theorem above one can replace the balls by arbitrary convex sets. To state our theorem first we have to define the meaning of 'shrinking a convex set'.

(C) 2008 Australian Mathematical Society 0004-9727/08 \$A2.00+0.00 
Let $M$ be an $n$-dimensional $(n \geq 2)$ complete and simply connected Riemannian manifold with sectional curvatures $\leq-1$. For a convex subset $C \subset M$ and a positive number $s>0$ we define the $s$-kernel of $C$ as

$$
C(s)=\{x \in C: B(x, s) \subset C\},
$$

where $B(x, s)$ denotes the open geodesic ball of radius $s$ and centre $x$. It is easy to see that the $s$-kernel is always closed, and possibly empty. Then we have the following result.

THEOREM. There is a constant $s_{0}>0$ such that for any $s>s_{0}$ and for any family of disjoint open convex sets $C_{n} \subset M, n \in A$, and for any point $x \in M-\cup_{n \in A} C_{n}$ there is a geodesic ray emanating from $x$ and avoiding $C_{n}(s)$ for all $n \in A$.

The constant $s_{0}$ is chosen such that the area of the geodesic ball $B\left(s_{0}\right)$ of radius $s_{0}$ in $\mathbb{H}^{2}(-1)$ is $2 \pi$. Since the area of

$$
B(r)=2 \pi(\cosh (r)-1)
$$

(see [2, Chapter 3]), this yields

$$
s_{0}=\cosh ^{-1}(2)<1.4
$$

which is slightly better than the constant given in [4].

To understand why such a statement may be true it is helpful to consider the following example. Let us assume that $M$ is a surface. Fix a point $p$ in $M$ and let us try to construct a counterexample, that is a family of disjoint open and convex sets not containing $p$ such that the $s$-kernels block all the rays from $p$. It is logical to try to find 'large' convex sets that block a lot of rays. The best candidates are half-spaces (one side of geodesics). Their $s$-kernels block the same rays as the half-spaces themselves. So let us assume that we have $n$ points $q_{1}, \ldots, q_{n}$, on the ideal boundary $S$ arranged in a cyclic order ( $\operatorname{set} q_{n+1}=q_{1}$ ) and let $\gamma_{i, j}$ be the geodesic connecting $q_{i}$ to $q_{j}$. Let $D$ be the connected component of $M-\bigcup_{i=1}^{n} \gamma_{i, i+1}$ that contains $p$ and assume that the boundary of $D$ contains all the geodesics $\gamma_{1,2}, \gamma_{2,3}, \ldots, \gamma_{n, 1}$. Set $C_{i}$ be the convex half-space bounded by $\gamma_{i, i+1}$ that does not contain $p$. Then with the exception of the rays $\gamma_{p, q_{i}}$ all the rays from $p$ are blocked by $C_{i}\left(s_{0}\right)$. To finish the counterexample we need to find disjoint convex sets in $D$ that will block the rays $\gamma_{p, q_{i}}, i=1,2, \ldots, n$. The smallest convex sets are geodesic balls; therefore we need to place a geodesic ball of radius $s_{0}$ (so that the $s_{0}$-kernels of the balls are not empty) centred on the rays $\gamma_{p, q_{i}}$, $i=1,2, \ldots, n$. But that is clearly impossible. From the Gauss-Bonnet theorem we have $\operatorname{Area}(D) \leq(n-2) \pi$. On the other hand the area comparison theorem (see for example [2, Theorem 3.7]) yields that the total area of the balls is at least $2 n \pi$.

As far as this example is concerned we could have chosen the constant $s_{0}$ such that $\operatorname{Area}\left(B\left(s_{0}\right)\right)=\pi$ in $\mathbb{H}^{2}(-1)$ but in the actual proof we need the larger constant. 


\section{Proof of the theorem}

Denote by $S$ the sphere at infinity (viewed as the set of equivalence classes of geodesic rays) and equip $M \cup S$ with the usual truncated cone topology. Then $S$ is homeomorphic to the sphere and $M \cup S$ is homeomorphic to the closed ball.

Define the projection map $\pi: M \cup S-\{p\} \rightarrow S$ such that $\pi(q)=\left[\gamma_{p q}\right]$, where $\gamma_{p q}$ denotes the geodesic ray starting from $p$ and passing through $q$, and for a geodesic ray $\gamma,[\gamma] \in S$ denotes the equivalence class. The following is easy to show.

Proposition 1. If $D \subset M$ is open, then $\pi(D)$ is an open set in $S$, and if $F \subset M$ is closed and bounded, then $\pi(F)$ is closed in $S$.

The proof is obvious.

We shall need the following technical definition. Let $p \in M$ be a fixed point and we call a subset $D \subset M \cup S-\{p\}$ a cone (with vertex $p$ ) if $q \in D$ implies that

$$
\gamma_{p q} \cup\left\{\left[\gamma_{p q}\right]\right\} \subset D \cup\{p\} .
$$

Since $p \in M$ will be fixed throughout the paper we shall sometimes call it a cone without referring to the vertex $p$.

Similarly for any set $D \subset M \cup S$ not containing $p$ we define the cone Cone $(D)$ of the set $D$ as

$$
\text { Cone }(D)=\left\{q \in M \cup S-\{p\}: \gamma_{p q} \cap D \neq \emptyset\right\} .
$$

Clearly the cone of an open set is also open. For of any set $D \subset M \cup S-\{p\}$ the cone naturally decomposes into three disjoint sets:

$$
\begin{gathered}
\operatorname{Cone}^{+}(D)=\{q \in \operatorname{Cone}(D):[p, q] \cap D=\emptyset\}, \\
\operatorname{Cone}^{-}(D)=\{q \in \operatorname{Cone}(D)-D:[p, q] \cap D \neq \emptyset\} \text { and } D .
\end{gathered}
$$

Here $[p, q]$ denotes the geodesic segment connecting $p$ and $q$.

Since the intersection of a cone and a convex set may not be convex or connected even if the cone is a cone over some convex set, the following technical proposition will be helpful.

Proposition 2. Let $D$ be a convex set not containing the point $p$ and $K$ be some cone based at $p$. Let

$$
D \cap K=\bigcup_{i \in A} D_{i},
$$

where $A$ is some index set and $D_{i}$ are the connected components of $D \cap K$. Then

$$
\pi\left(D_{i}\right) \cap \pi\left(D_{j}\right)=\emptyset,
$$

for all $i \neq j$. 
Proof. Suppose it is not true and

$$
\pi\left(D_{i}\right) \cap \pi\left(D_{j}\right) \neq \varnothing,
$$

for some $i$ and $j$. Let

$$
q \in \pi\left(D_{i}\right) \cap \pi\left(D_{j}\right)
$$

be any point and let $q_{i} \in D_{i}$ and $q_{j} \in D_{j}$ be points such that $\pi\left(q_{i}\right)=\pi\left(q_{j}\right)=q$. But then the geodesic segment $\left[q_{i}, q_{j}\right] \subset D \cap K$ and connects $D_{i}$ and $D_{j}$; therefore $D_{i} \cup D_{j}$ is connected and that is a contradiction.

The next proposition is also simple.

Proposition 3. Let $C \subset M$ be a convex open set not containing $p$, let $K$ be some cone with vertex $p$ and set $D=K \cap C$. Suppose there are points $x \in \mathrm{Cone}^{+}(D)$ and $y \in$ Cone $^{-}(D)$ such that there is a curve $\alpha:[0,1] \rightarrow \operatorname{Cone}(D)$ with $\alpha(0)=x$ and $\alpha(1)=y$. Then $\alpha$ intersects $D$.

PROOF. For simplicity denote by $[q, \infty)$ the infinite geodesic segment $\gamma_{p, q}-[p, q)$. Suppose $\alpha$ does not intersect $D$. Then we can sort the points of $[0,1]$ into two sets defined as follows:

$$
R_{1}=\{z \in[0,1]:[p, \alpha(z)] \cap D \neq \emptyset\} \quad \text { and } \quad R_{2}=\{z \in[0,1]:[\alpha(z), \infty] \cap D \neq \emptyset\} .
$$

From the definition above we have that $x \in R_{2}$ and $y \in R_{1}$. Since $\alpha([0,1]) \subset \operatorname{Cone}(D)$ it is clear that $R_{1} \cup R_{2}=[0,1]$. From the assumption that $\alpha([0,1]) \cap D=\emptyset$ and from the convexity of $D$ we obtain that $R_{1} \cap R_{2}=\emptyset$. Finally since $D$ is open (in the topology of $K$ ) and the conditions defining the sets $R_{1}$ and $R_{2}$ are open conditions we conclude that $R_{1}$ and $R_{2}$ are open subsets of $[0,1]$. But this contradicts the fact that $[0,1]$ is connected.

The following elementary lemma is crucial to the proof.

LEMMA 4. Let $M$ be an n-dimensional complete and simply connected Riemannian manifold with sectional curvatures $\leq-1$. Consider the geodesic triangle $\triangle_{p q r}$, where $p \in M$ and $q, r \in M \cup S$. Suppose that there is a point $x \in[p, q]$ such that $B\left(x, s_{0}\right) \cap[p, r]=\emptyset$. Then the geodesic segment $[r, q]$ intersects $B\left(x, s_{0}\right)$.

There are probably many different ways to prove this but let us argue in the spirit of the example given in the Introduction.

PROOF. For every $t \in[r, q]$ consider the segment $[p, t]$. The union of these segments is a ruled surface $F=\bigcup_{t \in[r, q]}[p, t]$. Since the second fundamental form of $F$ cannot be definite with respect to any normal, by the Gauss theorem we conclude that the sectional curvature of $F$ (with the metric inherited from $M$ ) is $\leq-1$.

Let us assume that on the contrary $[r, q] \cap B\left(x, s_{0}\right)=\emptyset$. Let

$$
B_{F}\left(x, s_{0}\right)=\left\{y \in F: \operatorname{dist}_{F}(x, y) \leq s_{0}\right\}
$$


be the geodesic ball of radius $s_{0}$ and centre $x$ on the surface $F$. Observe that

$$
B_{F}\left(x, s_{0}\right) \subset B\left(x, s_{0}\right) \cap F .
$$

Therefore we have $[r, q] \cap B_{F}\left(x, s_{0}\right)=\emptyset$, where the geodesic segment $[r, q]$ considered as a geodesic segment in $M$ is also a geodesic segment in $F$. From the Gauss-Bonnet formula applied for the surface $F$ we have that the $F$-area of the triangle $\triangle_{p q r}$ is less than $\pi$. On the other hand the triangle $\triangle_{p q r}$ contains a half-ball $B_{F}\left(x, s_{0}\right)$ whose $F$-area is greater than or equal to $\pi$ by the standard area comparison principle [3, Theorem 3.7] and that is a contradiction.

The following proposition also plays an important role.

PROpOSITION 5. Let $M$ be an n-dimensional complete and simply connected Riemannian manifold with sectional curvatures $\leq-1$. Let $C$ be an open convex set with $C(s) \neq \emptyset$ for some $s \geq s_{0}$. Suppose that there is a disjoint open convex set $D$ such that there is a point $q \in D$ with the property that the geodesic segment $[p, q]$ intersects $C(s)$. Then $\operatorname{dist}(p, D)>\operatorname{dist}(p, C)$.

PROOF. Suppose the statement is not true and let us assume that

$$
\operatorname{dist}(p, D) \leq \operatorname{dist}(p, C)
$$

Let $x \in[p, q] \cap C(s)$ be any point and $r \in \partial D$ be a point such that

$$
\operatorname{dist}(r) \leq \operatorname{dist}(p, C) .
$$

Consider the geodesic ball $B(x, s)$ and the cone Cone $(B(x, s))$. Then

$$
\text { either } \quad r \in \operatorname{Cone}(B(x, s)) \text { or } \quad r \notin \operatorname{Cone}(B(x, s)) \text {. }
$$

If $r \notin \operatorname{Cone}(B(x, s))$, then the geodesic segment $[p, r]$ does not intersect $B(x, s)$ and Lemma 4 implies that the geodesic segment $[r, q]$ intersects $B(x, s)$. But that is a contradiction since the geodesic segment $[r, q] \subset D$ and $B(x, s) \subset C$ and the sets $D, C$ were disjoint.

If $r \in \operatorname{Cone}(B(x, s))$ then $r \in \operatorname{Cone}^{+}(B(x, s))$ since

$$
\operatorname{dist}(p, r) \leq \operatorname{dist}(p, C) \leq \operatorname{dist}(p, B(x, s))
$$

and obviously $q \in \mathrm{Cone}^{-}(B(x, s))$. Now, if the geodesic segment $[r, q]$ $\subset$ Cone $(B(x, s))$, then by Proposition 3 we have that $[r, q]$ intersects $B(x, s)$ and the contradiction arises the same way as before. On the other hand if the geodesic segment $[r, q]$ leaves the cone then there is a point $r^{\prime} \in[r, q] \subset D$ such that $r^{\prime} \notin \operatorname{Cone}(B(x, s))$ and we can repeat the previous argument with $r^{\prime}$ in place of $r$ to obtain a contradiction. This concludes the proof of the proposition. 
Proof of TheOrem. First we show that it is enough to prove the theorem for finitely many bounded convex sets.

Let us assume on the contrary that we have a family $C_{n}, n \in A$, of disjoint open convex sets, a constant $s>s_{0}$ and a point $p \in M-\bigcup_{n \in A} C_{n}$ such that every geodesic ray emanating from $p$ intersects $C_{n}(s)$ for some $n \in A$.

Let $s_{0}<s^{\prime}<s$. Then clearly for any set $D$ and for any $q \in D(s)$,

$$
B\left(q, s-s^{\prime}\right) \subset D\left(s^{\prime}\right)
$$

Since

$$
\bigcup_{n \in A} \pi\left(C_{n}(s)\right)=S
$$

we have

$$
\bigcup_{q \in \cup\left\{C_{n}(s): n \in A\right\}} \pi\left(B\left(q, s-s^{\prime}\right)\right)=S .
$$

Since the projection of an open set is open and $S$ is compact there are finitely many points $q_{i}, i=1, \ldots, m$, such that $q_{i} \in C_{n}(s)$ for some $n \in A$ and

$$
\bigcup_{i=1}^{m} \pi\left(B\left(q_{i}, s-s^{\prime}\right)\right)=S .
$$

Let

$$
l=\max \left\{\operatorname{dist}\left(p, q_{i}\right): i=1, \ldots, m\right\}+s+1,
$$

and $A^{\prime} \subset A$ such that $n \in A^{\prime}$ if $q_{i} \in C_{n}(s)$ for some $i=1, \ldots, m$. Clearly $A^{\prime}$ is a finite subset of $A$.

For $n \in A^{\prime}$ set $C_{n}^{\prime}=C_{n} \cap B(p, l)$. Clearly $C_{n}^{\prime}$ is bounded open and convex, and if $q_{i} \in C_{n}(s)$, then $q_{i} \in C_{n}^{\prime}(s)$ as well. Therefore $B\left(q_{i}, s-s^{\prime}\right) \subset C_{n}^{\prime}\left(s^{\prime}\right)$ and

$$
\bigcup_{n \in A^{\prime}} \pi\left(C_{n}^{\prime}\left(s^{\prime}\right)\right)=S .
$$

To summarize: we have produced a finite family of disjoint bounded open convex sets $C_{n}^{\prime}, n \in A^{\prime}$, and a positive number $s_{0}<s^{\prime}$ such that every geodesic ray emanating from $p$ intersects $C_{n}^{\prime}\left(s^{\prime}\right)$ for some $n \in A^{\prime}$.

Therefore it is enough to show that the theorem is valid for such a family and from now on we assume that we have a finite family $C_{i}, i=1, \ldots, n$, of disjoint, bounded, open and convex sets, a constant $s>s_{0}$ and a point $p \in M-\bigcup_{i=1}^{n} C_{n}$ such that every geodesic ray emanating from $p$ intersects $C_{i}(s)$ for some $i=1, \ldots, n$, that is $\bigcup_{i=1}^{n} \pi\left(C_{i}(s)\right)=S$.

We are going to select different convex sets

$$
C_{a_{1}}, C_{a_{2}}, \ldots, C_{a_{i}}, \ldots
$$


from the family $\left\{C_{j}\right\}_{j=1}^{n}$ and construct subsets

$$
S \supset S_{1} \supset S_{2} \cdots \supset S_{i} \supset \cdots
$$

that are open and connected in the topology of $S$ such that the following properties are satisfied:

(1) $\pi\left(C_{a_{i}}(s)\right) \cap S_{i-1} \neq \emptyset$;

(2) $S_{i} \subset S_{i-1} \cap\left(\pi\left(C_{a_{i}}\right)-\pi\left(C_{a_{i}}(s)\right)\right)$ and there are points $q_{i} \in C_{a_{i}}(s)$ and $r_{i} \in \partial S_{i}$ such that $\pi\left(q_{i}\right) \in \partial S_{i}$ and $\left[p, r_{i}\right] \cap B\left(q_{i}, s\right)=\emptyset$;

(3) $D_{i}=C_{a_{i}} \cap \operatorname{Cone}\left(S_{i}\right)$ is open, $\operatorname{Cone}\left(D_{i}\right)=\operatorname{Cone}\left(S_{i}\right)$ and $\operatorname{Cone}^{+}\left(D_{i}\right)$ $\cap C_{j}(s)=\emptyset$ for all $j=1, \ldots, n$; and

(4) $S_{i} \cap \pi\left(C_{a_{j}}(s)\right)=\emptyset$ for all $j \leq i$.

Our goal is to show that this process never stops and this is a contradiction since we have only finitely many $C_{i}$ to select from.

We start by selecting the first set. Let $C_{a_{1}}$ be the set of the family $\left\{C_{j}\right\}_{j=1}^{n}$ with the smallest distance from $p$. Since the family is finite there has to be a set with the smallest distance, and if there is more than one set with minimal distance, choose one of them.

Next we construct the set $S_{1}$ satisfying the second property. Since $C_{a_{1}}$ is open and $C_{a_{1}}(s)$ is a closed bounded set from Proposition 1 we obtain that

$$
\pi\left(C_{a_{1}}\right)-\pi\left(C_{a_{1}}(s)\right)
$$

is open and since $S$ is locally connected all connected components are open as well. Let $S_{1}$ be a connected component of

$$
\pi\left(C_{a_{1}}\right)-\pi\left(C_{a_{1}}(s)\right)
$$

such that

$$
\partial\left(S_{1}\right) \cap \pi\left(C_{a_{1}}(s)\right) \neq \emptyset \quad \text { and } \quad \partial\left(S_{1}\right) \cap \partial \pi\left(C_{a_{1}}\right) \neq \emptyset .
$$

Naturally we have to show that such component exists.

To this end we show that if $S_{1}$ is any connected component of $\pi\left(C_{a_{1}}\right)-\pi\left(C_{a_{1}}(s)\right)$ we have $\partial S_{1} \cap \pi\left(C_{a_{1}}(s)\right) \neq \emptyset$. Suppose it is not true. Since $\pi\left(C_{a_{1}}(s)\right)$ is a closed subset of $S$ and therefore compact there is an open set $B \subset S$ such that $B \cap S_{1}=\emptyset$ and $\pi\left(C_{a_{1}}(s)\right) \subset B$. Since $S_{1}$ was a connected component of

$$
\pi\left(C_{a_{1}}\right)-\pi\left(C_{a_{1}}(s)\right),
$$

we have

$$
\left(\pi\left(C_{a_{1}}\right)-\pi\left(C_{a_{1}}(s)\right)\right)-S_{1}
$$

is also open, and therefore

$$
B^{\prime}=B \cup\left(\left(\pi\left(C_{a_{1}}\right)-\pi\left(C_{a_{1}}(s)\right)\right)-S_{1}\right)
$$


is open and disjoint from $S_{1}$. Since

$$
\pi\left(C_{a_{1}}\right) \subset B^{\prime} \cup S_{1}
$$

and $B^{\prime}$ and $S_{1}$ are disjoint we have decomposed $\pi\left(C_{a_{1}}\right)$ into the union of two disjoint open sets,

$$
\pi\left(C_{a_{1}}\right)=\left(\pi\left(C_{a_{1}}\right) \cap S_{1}\right) \cup\left(\pi\left(C_{a_{1}}\right) \cap B^{\prime}\right),
$$

and that is a contradiction since $\pi\left(C_{a_{1}}\right)$ as the projection of a connected set must be connected.

We also have to show that there is a connected component $S_{1}$ of

$$
\pi\left(C_{a_{1}}\right)-\pi\left(C_{a_{1}}(s)\right)
$$

such that

$$
\partial\left(S_{1}\right) \cap \partial \pi\left(C_{a_{1}}\right) \neq \varnothing .
$$

This is quite straightforward. Let $r_{1} \in \partial \pi\left(C_{a_{1}}\right)$ be any point. Since $C_{a_{1}}$ is bounded the infinite geodesic segment $\left[p, r_{1}\right]$ intersects the boundary of $C_{a_{1}}$, and if $x \in\left[p, r_{1}\right] \cap \partial C_{a_{1}}$ is an intersection point then there is an $\varepsilon>0$ such that

$$
\pi(B(x, \varepsilon)) \cap \pi C_{a_{1}}(s)=\emptyset \quad\left(C_{a_{1}}(s) \text { is a compact set }\right) .
$$

Then $B(x, \varepsilon) \cap C_{a_{1}}$ is open and convex, $r_{1} \in \partial \pi\left(B(x, \varepsilon) \cap C_{a_{1}}\right)$ and

$$
\pi\left(B(x, \varepsilon) \cap C_{a_{1}}\right) \subset \pi\left(C_{a_{1}}\right)-\pi\left(C_{a_{1}}(s)\right)
$$

is an open and connected set (the projection of a connected set is connected). Choose $S_{1}$ to be the connected component that contains $\pi\left(B(x, \varepsilon) \cap C_{a_{1}}\right)$.

Since

$$
\partial S_{1} \cap \pi\left(C_{a_{1}}(s)\right) \neq \emptyset
$$

let $q_{1} \in C_{a_{1}}(s)$ be any point such that

$$
\pi\left(q_{1}\right) \in \partial S_{1} \cap \pi\left(C_{a_{1}}(s)\right) .
$$

Such a point must exist because $C_{a_{1}}(s)$ is a compact set. Then $\left[p, r_{1}\right] \cap B\left(q_{1}, s\right)=\emptyset$ otherwise $r_{1}$ is in the interior of $\pi\left(B\left(q_{1}, s\right)\right)$, and since $B\left(q_{1}, s\right) \subset C_{a_{1}}$ the point $r_{1}$ would belong to the interior of $\pi\left(C_{a_{1}}\right)$ and that contradicts the choice of $r_{1}$. Therefore the set $S_{1}$ and the points $q_{1}, r_{1}$ satisfy the second property.

Set $D_{1}=C_{a_{1}} \cap \operatorname{Cone}\left(S_{1}\right)$. This is open since it is the intersection of two open sets, and since $S_{1} \subset \pi\left(C_{a_{1}}\right)$ one obtains that $\operatorname{Cone}\left(D_{1}\right)=\operatorname{Cone}\left(S_{1}\right)$. The important part is to show that $\operatorname{Cone}^{+}\left(D_{1}\right) \cap C_{j}(s)=\emptyset$ for all $j=1, \ldots, n$. Suppose it is not true and there is a $C_{j}$ such that $\operatorname{Cone}^{+}\left(D_{1}\right) \cap C_{j}(s) \neq \emptyset$. This means that there is 
a point $q \in D_{1} \subset C_{a_{1}}$ such that the geodesic segment $[p, q]$ intersects $C_{j}(s)$. Then Proposition 5 implies that $\operatorname{dist}\left(p, C_{a_{1}}\right)>\operatorname{dist}\left(p, C_{j}\right)$ but that is a contradiction since $C_{a_{1}}$ was chosen with minimal distance from $p$. This verifies the third property.

Finally the fourth property follows easily from the fact that

$$
S_{1} \subset \pi\left(C_{a_{1}}\right)-\pi\left(C_{a_{1}}(s)\right) .
$$

Let us now assume that we have chosen $C_{a_{1}}, \ldots, C_{a_{i}}$ and constructed the sets $S_{1}, \ldots, S_{i}$ satisfying the four properties. Let $A \subset\{1, \ldots, n\}$ be the set such that if $a \in A$, then

$$
\pi\left(C_{a}(s)\right) \cap S_{i} \neq \emptyset
$$

Since

$$
\bigcup_{i=1}^{n} \pi\left(C_{i}(s)\right)=S,
$$

the set $A \neq \emptyset$. Choose $C_{a_{i+1}}$ from the family $\left\{C_{a}\right\}_{a \in A}$ with the minimal distance from p. Again if there is more than one such set, choose one of them. It is clear that the first property is satisfied.

We now deal with the construction of $S_{i+1}$ and the verification of the second property. Let $C_{a_{i+1}}^{\prime}$ be a connected component of $C_{a_{i+1}} \cap \operatorname{Cone}\left(D_{i}\right)$ such that

$$
C_{a_{i+1}}(s) \cap C_{a_{i+1}}^{\prime} \neq \emptyset .
$$

Since $C_{a_{i+1}}^{\prime}$ is connected from Proposition 3 we obtain that

$$
C_{a_{i+1}}^{\prime} \subset \text { Cone }^{+}\left(D_{i}\right) \quad \text { or } \quad C_{a_{i+1}}^{\prime} \subset \text { Cone }^{-}\left(D_{i}\right) \text {. }
$$

Taking into consideration the third property we obtain that $C_{a_{i+1}}^{\prime} \subset \operatorname{Cone}^{-}\left(D_{i}\right)$.

The set $S_{i+1}$ is going to be a connected component of

$$
S_{i} \cap\left(\pi\left(C_{a_{i+1}}^{\prime}\right)-\pi\left(C_{a_{i+1}}(s)\right)\right) .
$$

First we show that $S_{i} \not \subset \pi\left(C_{a_{i+1}}^{\prime}\right)$. Suppose it is not true. Then there are points $q_{i} \in C_{a_{i}}(s)$ and

$$
r_{i} \in \partial S_{i}
$$

satisfying the second property and

$$
r_{i}, \pi\left(q_{i}\right) \in \mathrm{Cl}\left(\pi\left(C_{a_{i+1}}^{\prime}\right)\right) .
$$

Since $C_{a_{i+1}}^{\prime}$ is bounded one can find points

$$
q^{\prime} \in \mathrm{Cl}\left(C_{a_{i+1}}^{\prime}\right) \quad \text { and } \quad r^{\prime} \in \mathrm{Cl}\left(C_{a_{i+1}}^{\prime}\right)
$$


such that $\pi\left(q^{\prime}\right)=\pi\left(q_{i}\right)$ and $\pi\left(r^{\prime}\right)=\pi\left(r_{i}\right)$. Since $C_{a_{i+1}}^{\prime} \subset$ Cone $^{-}\left(D_{i}\right)$ we have that $q_{i} \in\left[p, q^{\prime}\right]$. By the second property the geodesic segment $\left[p, r^{\prime}\right] \subset\left[p, r_{i}\right]$ does not intersect the ball $B\left(q_{i}, s\right)$. Applying Lemma 4 we obtain that

$$
\left[r^{\prime}, q^{\prime}\right] \subset \mathrm{Cl}\left(C_{a_{i+1}}^{\prime}\right) \subset \mathrm{Cl}\left(C_{a_{i+1}}\right)
$$

intersects $B\left(q_{i}, s\right) \subset C_{a_{i}}$. Therefore the convex sets $C_{a_{i+1}}$ and $C_{a_{i}}$ intersect and that is a contradiction.

Let

$$
r \in S_{i}-\pi\left(C_{a_{i+1}}^{\prime}\right) \quad \text { and } \quad q \in C_{a_{i+1}}^{\prime} \cap C_{a_{i+1}}(s)
$$

be arbitrary points and let $\alpha:[0,1] \rightarrow S_{i}$ be a curve such that $\alpha(0)=r$ and $\alpha(1)$ $=\pi(q)$. Let

$$
t_{1}=\inf \left\{t \in[0,1]: \alpha([t, 1]) \subset \pi\left(C_{a_{i+1}}^{\prime}\right)\right\}
$$

and

$$
t_{2}=\inf \left\{t \in[0,1]: \alpha(t) \in \pi\left(C_{a_{i+1}}^{\prime} \cap C_{a_{i+1}}(s)\right) \text { and } \alpha([t, 1]) \subset \pi\left(C_{a_{i+1}}^{\prime}\right)\right\} .
$$

Since $\pi\left(C_{a_{i+1}}^{\prime}\right)$ is an open neighbourhood of $\pi\left(C_{a_{i+1}}^{\prime} \cap C_{a_{i+1}}(s)\right)$ in the topology of $S_{i}$ it is clear that $t_{1}<t_{2}$. Let $S_{i+1}$ be the connected component of

$$
S_{i} \bigcap\left(\pi\left(C_{a_{i+1}}^{\prime}\right)-\pi\left(C_{a_{i+1}}(s)\right)\right)
$$

that contains the curve $\alpha\left(t_{1}, t_{2}\right)$ and set $r_{i+1}=\alpha\left(t_{1}\right)$, and let

$$
q_{i+1} \in C_{a_{i+1}}^{\prime} \cap C_{a_{i+1}}(s)
$$

be any point such that $\pi\left(q_{i+1}\right)=\alpha\left(t_{2}\right)$. Such a point must exist since $C_{a_{i+1}}(s)$ is compact.

We must show now that the second property is satisfied with this choice. Clearly $S_{i+1}$ is open and connected in $S$. It is also clear that

$$
r_{i+1} \in \partial \pi\left(C_{a_{i+1}}^{\prime}\right),
$$

and therefore $r_{i+1} \in \partial S_{i+1}$. If

$$
B\left(q_{i+1}, s\right) \cap\left[p, r_{i+1}\right] \neq \emptyset,
$$

that would mean that

$$
r_{i+1} \in \pi\left(B\left(q_{i+1}, s\right)\right) .
$$

Denote by $D^{\prime}$ the connected component of $C_{a_{i+1}} \cap \operatorname{Cone}\left(D_{i}\right)$ such that $r_{i+1} \in \pi\left(D^{\prime}\right)$. Taking into account the fact that $D^{\prime}$ and therefore $\pi\left(D^{\prime}\right)$ are open we have that

$$
\pi\left(D^{\prime}\right) \cap \pi\left(C_{a_{i+1}}^{\prime}\right) \neq \emptyset
$$


Since by Proposition 5 the projections of different connected components must be disjoint we conclude that

$$
D^{\prime}=C_{a_{i+1}}^{\prime} \quad \text { and } \quad r_{i+1} \in \operatorname{Int}\left(C_{a_{i+1}}^{\prime}\right) .
$$

That contradicts the fact that $r_{i+1} \in \partial \pi\left(C_{a_{i+1}}^{\prime}\right)$.

We now move on to verification of the third property. Define the set

$$
D_{i+1}=\operatorname{Cone}\left(S_{i+1}\right) \cap C_{a_{i+1}} .
$$

Since by the construction $S_{i+1} \subset \pi\left(C_{a_{i+1}}\right)$,

$$
\text { Cone }\left(D_{i+1}\right)=\operatorname{Cone}\left(S_{i+1}\right) \text {. }
$$

Suppose there is a set $C_{j}$ such that

$$
\operatorname{Cone}^{+}\left(D_{i+1}\right) \cap C_{j}(s) \neq \emptyset .
$$

Then on the one hand by Proposition 5

$$
\operatorname{dist}\left(p, C_{a_{i+1}}\right)>\operatorname{dist}\left(p, C_{j}\right),
$$

and on the other hand

$$
\pi\left(C_{j}(s)\right) \cap S_{i} \neq \emptyset .
$$

Therefore $j \in A$ and this is a contradiction since $C_{a_{i+1}}$ was chosen out of the sets $\left\{C_{a}\right\}_{a \in A}$ with the minimum distance.

Now we verify the fourth property. The set $S_{i+1}$ was constructed to be a connected component of

$$
S_{i} \bigcap\left(\pi\left(C_{a_{i+1}}^{\prime}\right)-\pi\left(C_{a_{i+1}}(s)\right)\right),
$$

which implies that

$$
S_{i+1} \cap \pi\left(C_{a_{i+1}}(s)\right)=\emptyset .
$$

On the other hand since $S_{i+1} \subset S_{j}$ for $j \leq i$ and from the fourth property we know that

$$
S_{j} \cap \pi\left(C_{a_{j}}(s)\right)=\emptyset,
$$

for all $j \leq i$ we obtain

$$
S_{i+1} \cap \pi\left(C_{a_{j}}(s)\right)=\emptyset,
$$

for all $j \leq i+1$.

Finally, combining the first and the last properties one obtains that the selected sets are all different. This shows that the construction never stops, which is a contradiction and the proof of the theorem is complete. 


\section{References}

[1] S. Buyalo, V. Schroeder and M. Walz, 'Geodesics avoiding subsets in surfaces of negative curvature', Ergod. Th. \& Dynam. Sys. 20 (2000), 991-1006.

[2] I. Chavel, Riemannian Geometry - A Modern Introduction (Cambridge University Press, Cambridge, 1993).

[3] J. Parkkonen and F. Paulin, Geom. Funct. Anal. 15 (2005), 491-533.

[4] _ ' 'Prescribing the behavior of geodesics in negative curvature', Preprint, 2007, arXiv:07062579v1.

[5] V. Schroeder, 'Bounded geodesics in manifolds of negative curvature', Math. Z. 235 (2000), $817-$ 828.

[6] M. Walz, 'Invariant subsets of the geodesic flow on negatively curved manifolds', Thesis, Zurich, 1998.

ALBERT BORBÉLY, Kuwait University, Faculty of Science,

Department of Mathematics and Computer Science, P.O. Box 5969, Safat 13060, Kuwait

e-mail: borbely@mcs.sci.kuniv.edu.kw 